\title{
Lyapunov-stable discrete-time model reference adaptive control
}

\author{
S. Akhtar*, ${ }^{*}$ and D. S. Bernstein \\ Department of Aerospace Engineering, The University of Michigan, Ann Arbor, MI 48109-2140, U.S.A.
}

\begin{abstract}
SUMMARY
Discrete-time model reference adaptive control (MRAC) is considered with both least squares and projection algorithm parameter identification. For both cases complete Lyapunov proofs are given for stability and convergence. The results extend the approach of Johansson (Int. J. Control 1989; 50(3): 859-869) to include Lyapunov stability for MRAC when the normalized projection algorithm is used for parameter identification. Copyright (C) 2005 John Wiley \& Sons, Ltd.
\end{abstract}

KEY WORDS: model reference adaptive control; discrete-time; Lyapunov stable

\section{INTRODUCTION}

In model reference adaptive control (MRAC), the objective is to have the plant emulate the dynamics of a specified model in response to a family of command signals. Model reference adaptive control has been extensively developed for continuous-time systems [1] and discretetime systems [2], where the boundedness of the controller parameters and the convergence of the tracking error are demonstrated using the Gronwall-Bellman lemma and the key technical lemma, respectively. The objective of the present paper is to unify and extend discrete-time MRAC by constructing Lyapunov functions for error convergence and Lyapunov stability.

Discrete-time MRAC algorithms have been based on a variety of parameter identification algorithms. In particular, the recursive least squares (RLS) algorithm and the projection algorithm are used in Reference [3], where convergence is based on the key technical lemma. This method of proof yields convergence but does not imply Lyapunov stability of the error system. MRAC is considered in the presence of additive noise in References [2,4-6]. In these results, convergence of the tracking error and parameters is guaranteed almost surely, but stochastic Lyapunov stability is not demonstrated.

Lyapunov stability of discrete-time MRAC and convergence of the error to a finite set is demonstrated in Reference [7], where the RLS algorithm is used for parameter identification and

\footnotetext{
*Correspondence to: S. Akhtar, Department of Aerospace Engineering, The University of Michigan, Ann Arbor, MI 48109-2140, U.S.A.

${ }^{\dagger}$ E-mail: akhtars@umich.edu

Contract/grant sponsor: Air Force Office of Scientific Research; contract/grant number: F49620-01-1-0094
}

Copyright (C) 2005 John Wiley \& Sons, Ltd.

Received 11 March 2004

Revised 7 September 2004

Accepted 20 April 2005 
a Lyapunov candidate is applied to the time-varying error system. Stochastic Lyapunov stability of MRAC is addressed [8].

The novel Lyapunov construction of [7-10] is of independent interest since it involves the logarithm of a quadratic form. A similar construction is used in Reference [11] for full-statefeedback adaptive stabilization and in Reference [12] to a more general class of direct adaptive stabilization algorithms with normalized adaptive laws.

In view of these developments, the present paper has the following objectives. First, we refine the proof of Lyapunov stability and convergence for RLS parameter identification, where we account for the time-varying dynamics of the error system and clarify the assumptions under which the construction is valid. Next, we construct a Lyapunov proof of MRAC for the projection algorithm. These constructs remove the need for the key technical lemma used in Reference [3]. Finally, we simulate both MRAC algorithms to demonstrate their performance.

The contents of the paper are as follows. In Section 2 we present the solution to the model matching control problem in the case of a known plant. In Section 3 the model matching error dynamics in the case of an unknown plant are formulated. An adaptive control law with RLS parameter identification is derived in Section 4. Stability of RLS adaptive control is demonstrated in Section 5. An adaptive control law with the projection algorithm for parameter identification is presented in Section 6, and a proof of stability is given in Section 7. Section 8 presents simulation results. Finally, some concluding remarks on future extensions are made in Section 9.

\section{MODEL REFERENCE CONTROL FOR A KNOWN PLANT}

Consider a SISO process described by the DARMA model

$$
y(k)=-\sum_{i=1}^{n} a_{i} y(k-i)+\sum_{j=0}^{m} b_{j} u(k-j), \quad k \geqslant 0
$$

The model (1) can be written in terms of the forward shift operator $\mathbf{q}$ as

$$
\mathbf{A}(\mathbf{q}) y(k)=\mathbf{B}(\mathbf{q}) u(k)
$$

where $\mathbf{A}$ and $\mathbf{B}$ are polynomials of degree $n$ and $m$, respectively, defined by

$$
\mathbf{A}(\mathbf{q}) \triangleq \mathbf{q}^{n}+a_{1} \mathbf{q}^{n-1}+\cdots+a_{n}
$$

and

$$
\mathbf{B}(\mathbf{q}) \triangleq b_{0} \mathbf{q}^{m}+b_{1} \mathbf{q}^{m-1}+\cdots+b_{m}
$$

where $b_{0} \neq 0$. We define the delay $d \triangleq n-m$ and make the following assumptions about the plant.

Assumption 2.1

The realization (2) is minimal, i.e. $\mathbf{A}$ and $\mathbf{B}$ are coprime.

Assumption 2.2

All roots of $\mathbf{B}(\mathbf{q})$ are inside the unit circle. 
Assumption 2.3

$n$ and $m$ are known, and $m<n$.

Assumption 2.4

$b_{0}$ is known.

To modify the dynamics (2) we consider the 2-DOF model matching control law

$$
u(k)=\frac{\mathbf{T}(\mathbf{q})}{\mathbf{R}(\mathbf{q})} u_{\mathrm{c}}(k)-\frac{\mathbf{S}(\mathbf{q})}{\mathbf{R}(\mathbf{q})} y(k)
$$

where $u_{\mathrm{c}}$ is the command signal. The closed-loop system (2)-(5) (see Figure 1) is then given by

$$
y(k)=\frac{\mathbf{B}(\mathbf{q}) \mathbf{T}(\mathbf{q})}{\mathbf{A}(\mathbf{q}) \mathbf{R}(\mathbf{q})+\mathbf{B}(\mathbf{q}) \mathbf{S}(\mathbf{q})} u_{\mathrm{c}}(k)
$$

We want the response from the command signal $u_{\mathrm{c}}$ to the output $y$ to be described by the reference model

$$
y_{\mathrm{m}}(k)=\frac{\mathbf{B}_{\mathrm{m}}(\mathbf{q})}{\mathbf{A}_{\mathrm{m}}(\mathbf{q})} u_{\mathrm{c}}(k)
$$

We make the following assumptions about the reference model.

Assumption 2.5

$\mathbf{A}_{\mathrm{m}}(\mathbf{q})$ is monic and stable.

Assumption 2.6

$\operatorname{deg} \mathbf{A}_{\mathrm{m}}(\mathbf{q})-\operatorname{deg} \mathbf{B}_{\mathrm{m}}(\mathbf{q})=d$, i.e. the reference model has the same delay as the plant.

The closed-loop system (6) and the reference model (7) have the same forced response if

$$
\frac{\mathbf{B}(\mathbf{q}) \mathbf{T}(\mathbf{q})}{\mathbf{A}(\mathbf{q}) \mathbf{R}(\mathbf{q})+\mathbf{B}(\mathbf{q}) \mathbf{S}(\mathbf{q})}=\frac{\mathbf{B}_{\mathrm{m}}(\mathbf{q})}{\mathbf{A}_{\mathrm{m}}(\mathbf{q})}
$$

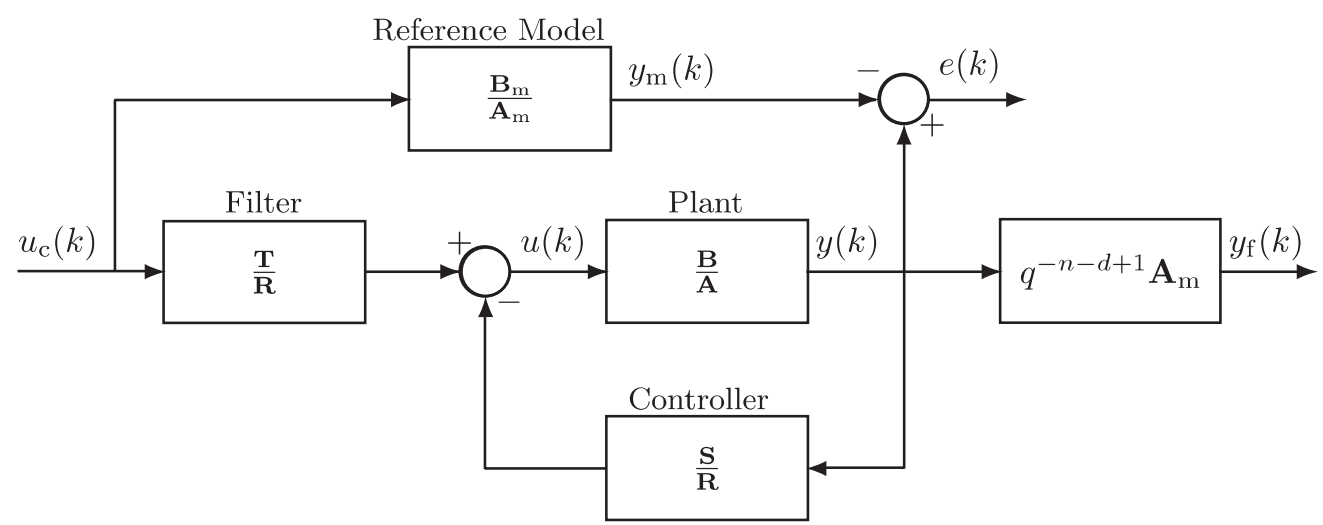

Figure 1. Model reference control. 
which is equivalent to

$$
\frac{\mathbf{T}(\mathbf{q})}{\mathbf{A}(\mathbf{q}) \mathbf{R}(\mathbf{q})+\mathbf{B}(\mathbf{q}) \mathbf{S}(\mathbf{q})}=\frac{\mathbf{B}_{\mathrm{m}}(\mathbf{q})}{\mathbf{A}_{\mathrm{m}}(\mathbf{q}) \mathbf{B}(\mathbf{q})}
$$

The roots of the closed-loop characteristic polynomial $\mathbf{A}_{\mathrm{m}}(\mathbf{q}) \mathbf{B}(\mathbf{q})$ consist of the roots of $\mathbf{A}_{\mathrm{m}}(\mathbf{q})$ as well as the roots of $\mathbf{B}(\mathbf{q})$, all of which are stable by assumption. Let $n_{\mathrm{m}} \triangleq \operatorname{deg} \mathbf{A}_{\mathrm{m}}(\mathbf{q})$ and define

$$
\mathbf{P}(\mathbf{q}) \triangleq \mathbf{A}_{\mathrm{m}}(\mathbf{q}) \mathbf{B}(\mathbf{q})=b_{0} \mathbf{q}^{\left(n_{\mathrm{m}}+m\right)}+p_{1} \mathbf{q}^{\left(n_{\mathrm{m}}+m\right)-1}+\cdots+p_{n_{\mathrm{m}}+m}
$$

To satisfy (9) it suffices to choose

$$
\mathbf{T}(\mathbf{q})=\mathbf{B}_{\mathrm{m}}(\mathbf{q})
$$

and require that $\mathbf{R}(\mathbf{q})$ and $\mathbf{S}(\mathbf{q})$ satisfy

$$
\mathbf{A}(\mathbf{q}) \mathbf{R}(\mathbf{q})+\mathbf{B}(\mathbf{q}) \mathbf{S}(\mathbf{q})=\mathbf{P}(\mathbf{q})
$$

Defining $n_{\mathbf{R}} \triangleq \operatorname{deg} \mathbf{R}(\mathbf{q}), n_{\mathbf{S}} \triangleq \operatorname{deg} \mathbf{S}(\mathbf{q}), n_{\mathrm{e}} \triangleq n+n_{\mathbf{R}}+1$, and $n_{u} \triangleq n_{\mathbf{R}}+n_{\mathbf{S}}+2$, (12) can be written as

$$
M\left[\begin{array}{c}
\mathscr{C}(\mathbf{R}) \\
\mathscr{C}(\mathbf{S})
\end{array}\right]=\mathscr{C}(\mathbf{P})
$$

where $M \in \mathbb{R}^{n_{\mathrm{e}} \times n_{u}}$ is the Sylvester matrix

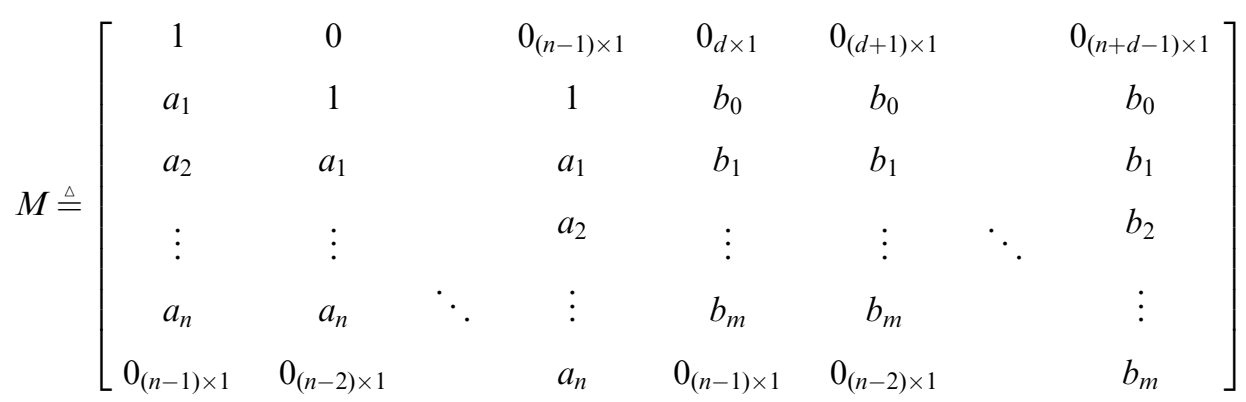

Furthermore, $\quad \mathscr{C}(\mathbf{R})=\left[\begin{array}{llll}r_{0} & r_{1} & \cdots & r_{n_{\mathbf{R}}}\end{array}\right]^{\mathrm{T}}, \quad \mathscr{C}(\mathbf{S})=\left[\begin{array}{llll}s_{0} & s_{1} & \cdots & s_{n_{\mathbf{S}}}\end{array}\right]^{\mathrm{T}}, \quad$ and $\quad \mathscr{C}(\mathbf{P})=$ $\left[\begin{array}{llll}b_{0} & p_{1} & \cdots & p_{n_{\mathrm{m}}+m}\end{array}\right]^{\mathrm{T}}$ are vectors containing the coefficients of $\mathbf{R}(\mathbf{q}), \mathbf{S}(\mathbf{q})$, and $\mathbf{P}(\mathbf{q})$, respectively. In the remainder of the paper, we omit the explicit dependence of polynomial operators on $\mathbf{q}$.

Proposition 2.1

Assume that $n_{\mathrm{m}}=2 n-m-1$ and $n_{\mathbf{S}}=n-1$. Then, for each $n_{\mathbf{R}} \geqslant 0$ there exist unique polynomials $\mathbf{R}$ and $\mathbf{S}$ satisfying (13), or equivalently (12). Furthermore, if $n_{\mathbf{R}} \geqslant n_{\mathbf{S}}$ then the control law (5) is causal.

Proof

Since $n_{\mathbf{S}}=n-1$ it follows that $M \in \mathbb{R}^{\left(n+n_{\mathbf{R}}+1\right) \times\left(n+n_{\mathbf{R}}+1\right)}$ is square. Also, since $\mathbf{A}$ and $\mathbf{B}$ are relatively coprime, it follows that $M$ is non-singular and the solution $[\mathscr{C}(\mathbf{R}) \quad \mathscr{C}(\mathbf{S})]^{\mathrm{T}}$ to (13) is unique. From (11) we have

$$
\operatorname{deg} \mathbf{T}=\operatorname{deg} \mathbf{B}_{\mathrm{m}}=\operatorname{deg} \mathbf{A}_{\mathrm{m}}-d=2 n-m-1-n+m=n-1
$$


Finally if $n_{\mathbf{R}} \geqslant n_{\mathbf{S}}$, then $\operatorname{deg} \mathbf{R} \geqslant \operatorname{deg} \mathbf{S}=\operatorname{deg} \mathbf{T}$, and thus the model matching controller (5) is causal.

Henceforth in accordance with Proposition 2.1 we assume that $\operatorname{deg} \mathbf{S}=n-1$ and $\operatorname{deg} \mathbf{A}_{\mathrm{m}}=2 n-m-1$ so that $\operatorname{deg} \mathbf{P}=2 n-1$. Also, to obtain a minimum degree causal controller we assume that $\operatorname{deg} \mathbf{R}=n-1$ so that $M \in \mathbb{R}^{2 n \times 2 n}$. Hence we write

$$
\mathbf{R} \triangleq r_{0} \mathbf{q}^{n-1}+r_{1} \mathbf{q}^{n-2}+\cdots+r_{n-1}
$$

and

$$
\mathbf{S} \triangleq s_{0} \mathbf{q}^{n-1}+s_{1} \mathbf{q}^{n-2}+\cdots+s_{n-1}
$$

where $r_{0}$ and $s_{0}$ are non-zero. In fact, it follows from (12) and (15) that $r_{0}=b_{0}$.

Next to obtain a linear estimation model in terms of the controller we define the filtered output signal (see Figure 1)

$$
y_{\mathrm{f}}(k) \triangleq \mathbf{q}^{-n-d+1} \mathbf{A}_{\mathrm{m}} y(k)
$$

Then it follows from (2) that

$$
y_{\mathrm{f}}(k)=\frac{\mathbf{q}^{-n-d+1} \mathbf{A}_{\mathrm{m}} \mathbf{B}}{\mathbf{A}} u(k)
$$

With the model matching condition (12), $y_{\mathrm{f}}$ satisfies

$$
\begin{aligned}
y_{\mathrm{f}}(k+d) & =\frac{\mathbf{q}^{-n+1}(\mathbf{A} \mathbf{R}+\mathbf{B S})}{\mathbf{A}} u(k) \\
& =\left(\mathbf{R}+\mathbf{S} \frac{\mathbf{B}}{\mathbf{A}}\right) u(k-n+1) \\
& =\mathbf{R} u(k-n+1)+\mathbf{S} y(k-n+1)
\end{aligned}
$$

Since $r_{0}=b_{0}(19)$ can be written as the linear identification model

$$
y_{\mathrm{f}}(k+d)=b_{0} u(k)+\varphi^{\mathrm{T}}(k) \theta
$$

where the parameter vector $\theta$ and the regressor $\varphi(k)$ are defined by

$$
\theta \triangleq\left[\begin{array}{llllll}
r_{1} & \cdots & r_{n-1} & s_{0} & \cdots & s_{n-1}
\end{array}\right]^{\mathrm{T}} \in \mathbb{R}^{2 n-1}
$$

and

$$
\varphi(k) \triangleq\left[\begin{array}{llllll}
u(k-1) & \cdots & u(k-n+1) \quad y(k) & \cdots & y(k-n+1)
\end{array}\right]^{\mathrm{T}} \in \mathbb{R}^{2 n-1}
$$

Using (19) and (20) the model matching control law (5) can be written as

$$
u(k)=-\frac{1}{b_{0}}\left[\varphi^{\mathrm{T}}(k) \theta-\mathbf{q}^{-n+1} \mathbf{B}_{\mathrm{m}} u_{\mathrm{c}}(k)\right]
$$

The filtered plant model (20) and the control law (23) are now in a form suitable for direct adaptive control. 


\section{MODEL MATCHING ERROR DYNAMICS}

When the plant (2) is unknown we cannot solve (13) for the controller parameters $\mathbf{R}$ and $\mathbf{S}$. Hence, let $\hat{\mathbf{R}}(k)$ and $\hat{\mathbf{S}}(k)$ be polynomials in $\mathbf{q}$ that are estimates of $\mathbf{R}$ and $\mathbf{S}$ at time $k$. Then in place of (5), the estimated model matching controller is

$$
u(k)=\frac{\mathbf{B}_{\mathrm{m}}}{\hat{\mathbf{R}}(k)} u_{\mathrm{c}}(k)-\frac{\hat{\mathbf{S}}(k)}{\hat{\mathbf{R}}(k)} y(k)
$$

With (24) the closed-loop system has the form

$$
y(k)=\frac{\mathbf{B B}_{\mathrm{m}}}{\mathbf{A} \hat{\mathbf{R}}(k)+\mathbf{B} \hat{\mathbf{S}}(k)} u_{\mathrm{c}}(k)
$$

Next let $\hat{\theta}(k)$ denote an estimate of $\theta$ at time $k$ and define the parameter error

$$
\tilde{\theta}(k) \triangleq \hat{\theta}(k)-\theta
$$

and the filtered output error signal (see Figure 2)

$$
e_{\mathrm{f}}(k) \triangleq y_{\mathrm{f}}(k)-\mathbf{q}^{-n-d+1} \mathbf{B}_{\mathrm{m}} u_{\mathrm{c}}(k)
$$

To express $e_{\mathrm{f}}(k)$ in terms of $\tilde{\theta}$, note that

$$
\begin{aligned}
y_{\mathrm{f}}(k+d) & =\mathbf{q}^{-n+1} \frac{\mathbf{A}_{\mathrm{m}} \mathbf{B B}_{\mathrm{m}}}{\mathbf{A} \hat{\mathbf{R}}(k)+\mathbf{B} \hat{\mathbf{S}}(k)} u_{\mathrm{c}}(k)=\mathbf{q}^{-n+1} \frac{\mathbf{A R}+\mathbf{B S}}{\mathbf{A} \hat{\mathbf{R}}(k)+\mathbf{B} \hat{\mathbf{S}}(k)} \mathbf{B}_{\mathrm{m}} u_{\mathrm{c}}(k) \\
& =\mathbf{q}^{-n+1} \frac{\mathbf{R}+\frac{\mathbf{B S}}{\mathbf{A}}}{\hat{\mathbf{R}}(k)+\frac{\mathbf{B} \hat{\mathbf{S}}(k)}{\mathbf{A}}} \mathbf{B}_{\mathrm{m}} u_{\mathrm{c}}(k)=\mathbf{q}^{-n+1} \frac{\mathbf{R} u(k)+\mathbf{S} y(k)}{\hat{\mathbf{R}}(k) u(k)+\hat{\mathbf{S}}(k) y(k)} \mathbf{B}_{\mathrm{m}} u_{\mathrm{c}}(k) \\
& =\frac{b_{0} u(k)+\varphi^{\mathrm{T}}(k) \theta}{\mathbf{q}^{n-1}\left[b_{0} u(k)+\varphi^{\mathrm{T}}(k) \hat{\theta}(k)\right]} \mathbf{B}_{\mathrm{m}} u_{\mathrm{c}}(k)
\end{aligned}
$$

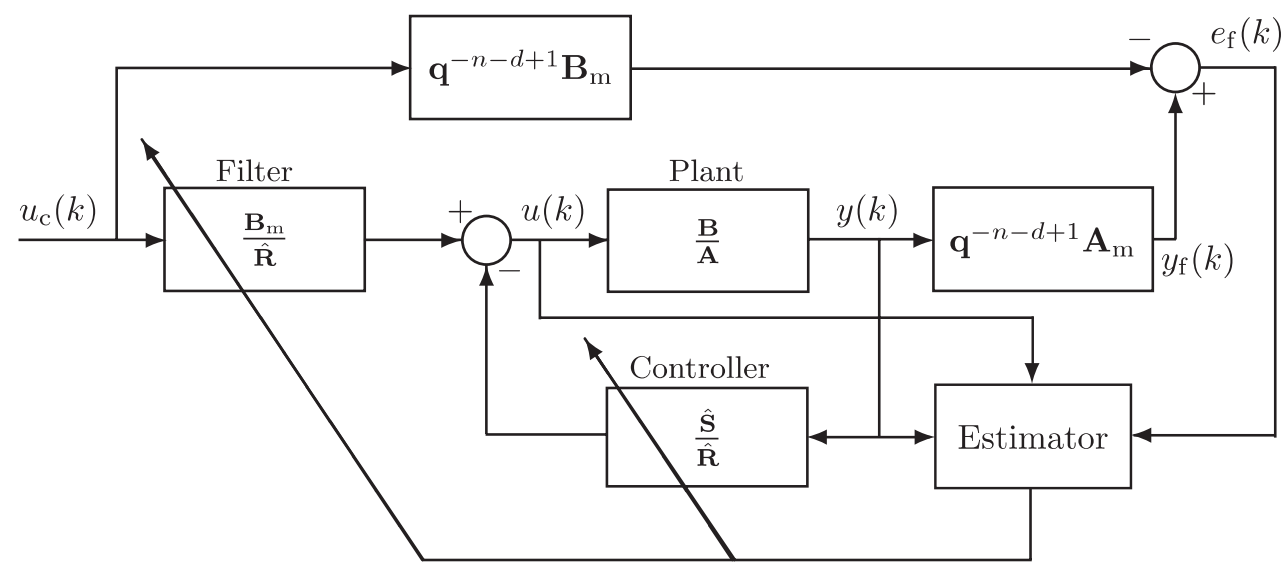

Figure 2. Model reference adaptive control block diagram. 
Combining (20) and (28) yields

$$
b_{0} u(k)+\varphi^{\mathrm{T}}(k) \hat{\theta}(k)=\mathbf{q}^{-n+1} \mathbf{B}_{\mathrm{m}} u_{\mathrm{c}}(k)
$$

From (20), (27) and (29) it follows that

$$
\begin{aligned}
e_{\mathrm{f}}(k+d) & =y_{\mathrm{f}}(k+d)-\mathbf{q}^{-n+1} \mathbf{B}_{\mathrm{m}} u_{\mathrm{c}}(k) \\
& =b_{0} u(k)+\varphi^{\mathrm{T}}(k) \theta-b_{0} u(k)-\varphi^{\mathrm{T}}(k) \hat{\theta}(k) \\
& =-\varphi^{\mathrm{T}}(k) \tilde{\theta}(k)
\end{aligned}
$$

To formulate the model matching error dynamics we note that the plant (2) can be written in the $n$th order fraction form as [13] (see Figure 3(a))

$$
\begin{aligned}
& \mathbf{A} \xi(k-n)=u(k) \\
& y(k)=\mathbf{B} \xi(k-n)
\end{aligned}
$$

From (17) and (32) it follows that

$$
y_{\mathrm{f}}(k+d)=\mathbf{q}^{-n+1} \mathbf{A}_{\mathrm{m}} y(k)=\mathbf{q}^{-n+1} \mathbf{A}_{\mathrm{m}} \mathbf{B} \xi(k-n)=\mathbf{q}^{-2 n+1} \mathbf{P} \xi(k)
$$

In a similar manner the reference model can be written in the $2 n-1$ th order non-minimal fraction form (see Figure 3(b))

$$
\begin{gathered}
\mathbf{A}_{\mathrm{m}} \mathbf{B} \xi_{\mathrm{m}}(k-n)=\mathbf{B}_{\mathrm{m}} u_{\mathrm{c}}(k) \\
y_{\mathrm{m}}(k)=\mathbf{B} \xi_{\mathrm{m}}(k-n)
\end{gathered}
$$

From (34) it follows that

$$
\mathbf{q}^{-n+1} \mathbf{B}_{\mathrm{m}} u_{\mathrm{c}}(k)=\mathbf{q}^{-n+1} \mathbf{A}_{\mathrm{m}} \mathbf{B} \xi_{\mathrm{m}}(k-n)=\mathbf{q}^{-2 n+1} \mathbf{P} \xi_{\mathrm{m}}(k)
$$

Using (33) and (36), the $d$-step ahead filtered output error can now be written as

$$
\begin{aligned}
e_{\mathrm{f}}(k+d) & =y_{\mathrm{f}}(k+d)-\mathbf{q}^{-n+1} \mathbf{B}_{\mathrm{m}} u_{\mathrm{c}}(k) \\
& =\mathbf{q}^{-2 n+1} \mathbf{P}\left[\xi(k)-\xi_{\mathrm{m}}(k)\right] \\
& =\mathbf{q}^{-2 n+1} \mathbf{P} \xi_{\mathrm{e}}(k)
\end{aligned}
$$

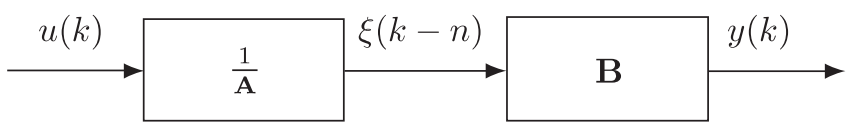

(a)

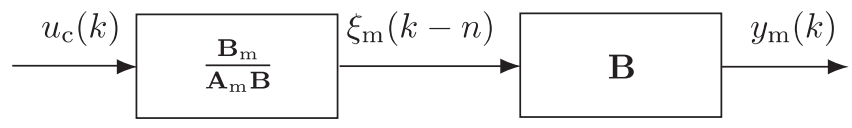

(b)

Figure 3. Fraction forms of the plant and the reference model. 
where

$$
\xi_{\mathrm{e}}(k) \triangleq \xi(k)-\xi_{\mathrm{m}}(k)
$$

Next define plant, reference model, and model-matching error states by

$$
\begin{gathered}
x(k) \triangleq\left[\begin{array}{lll}
\xi(k-1) & \cdots & \xi(k-2 n+1)
\end{array}\right]^{\mathrm{T}} \in \mathbb{R}^{2 n-1} \\
x_{\mathrm{m}}(k) \triangleq\left[\begin{array}{lll}
\xi_{\mathrm{m}}(k-1) & \cdots & \xi_{\mathrm{m}}(k-2 n+1)
\end{array}\right]^{\mathrm{T}} \in \mathbb{R}^{2 n-1}
\end{gathered}
$$

and

$$
x_{\mathrm{e}}(k) \triangleq x(k)-x_{\mathrm{m}}(k)
$$

Then

$$
x_{\mathrm{e}}(k)=\left[\begin{array}{lll}
\xi_{\mathrm{e}}(k-1) & \cdots & \xi_{\mathrm{e}}(k-2 n+1)
\end{array}\right]^{\mathrm{T}} \in \mathbb{R}^{2 n-1}
$$

Since

$$
\begin{aligned}
\xi_{\mathrm{e}}(k) & =\mathbf{q} \xi_{\mathrm{e}}(k-1) \\
& =-\mathbf{q}\left[\frac{1}{b_{0}} q^{-2 n+1} \mathbf{P}-1\right] \xi_{\mathrm{e}}(k-1)+\frac{1}{b_{0}} q^{-2 n+1} \mathbf{P} \xi_{\mathrm{e}}(k) \\
& =-\mathbf{q}\left[\frac{1}{b_{0}} q^{-2 n+1} \mathbf{P}-1\right] \xi_{\mathrm{e}}(k-1)+\frac{1}{b_{0}} e_{\mathrm{f}}(k+d)
\end{aligned}
$$

a state equation for $x_{\mathrm{e}}$ in controllable canonical form is given by

$$
x_{\mathrm{e}}(k+1)=A x_{\mathrm{e}}(k)+\frac{1}{b_{0}} B e_{\mathrm{f}}(k+d), \quad k \geqslant 0
$$

where

$$
A \triangleq\left[\begin{array}{ccc}
-p_{1} / b_{0} & \cdots & -p_{2 n-1} / b_{0} \\
\hline I_{(2 n-2) \times(2 n-2)} & \vdots \\
& 0
\end{array}\right], \quad B \triangleq\left[\begin{array}{c}
1 \\
0 \\
\vdots \\
0
\end{array}\right]
$$

Note that $A$ is asymptotically stable. Alternatively, using (30) the model matching dynamics (44) can be written as

$$
x_{\mathrm{e}}(k+1)=A x_{\mathrm{e}}(k)-\frac{1}{b_{0}} B \varphi^{\mathrm{T}}(k) \tilde{\theta}(k), \quad k \geqslant 0
$$

Next we show that the state $x$ defined in (39) is related to $\varphi$ through a non-singular transformation. 
Lemma 3.1

The plant state $x$ defined by (39) and the regressor (22) are related by

$$
\varphi(k)=M_{0} x(k)
$$

where the non-singular matrix $M_{0} \in \mathbb{R}^{(2 n-1) \times(2 n-1)}$ is defined by

$$
M_{0} \triangleq\left[\begin{array}{cccccccccc}
1 & a_{1} & a_{2} & \cdots & a_{n} & & & 0_{1 \times(n-2)} & \\
& \ddots & & & \ddots & & \ddots & \\
& & & & & & & & & \\
& 0_{1 \times(n-2)} & & & & a_{1} & a_{2} & \cdots & a_{n} \\
0_{1 \times(d-1)} b_{0} & b_{1} & \cdots & b_{m} & & & 0_{1 \times(n-1)} & \\
& \ddots & & & \ddots & & \ddots & \\
& 0_{1 \times(n+d-2)} & & & & b_{0} & b_{1} & \cdots & b_{m}
\end{array}\right]
$$

Proof

It follows from (31) and (32) that

$$
\varphi(k)=\left[\begin{array}{c}
u(k-1) \\
u \\
u(k-n+1) \\
y(k) \\
\vdots \\
y(k-n+1)
\end{array}\right]=\left[\begin{array}{c}
\mathbf{A}(\mathbf{q}) \xi(k-n-1) \\
\vdots \\
\mathbf{A}(\mathbf{q}) \xi(k-2 n+1) \\
\mathbf{B}(\mathbf{q}) \xi(k-n) \\
\vdots \\
\mathbf{B}(\mathbf{q}) \xi(k-2 n+1)
\end{array}\right]
$$

Equations in (49) can be written as

$$
\begin{aligned}
& u(k-1)=\mathbf{A}(\mathbf{q}) \xi(k-n-1) \\
& =\left[\mathbf{q}^{n}+a_{1} \mathbf{q}^{n-1}+\cdots+a_{n}\right] \xi(k-n-1) \\
& =\xi(k-1)+a_{1} \xi(k-2)+\cdots+a_{n} \xi(k-n-1) \\
& =\left[\begin{array}{llll}
1 & a_{1} & \cdots & a_{n}
\end{array}\right]\left[\begin{array}{llll}
\xi(k-1) & \cdots & \xi(k-n-1)
\end{array}\right]^{\mathrm{T}} \\
& \vdots \\
& u(k-n+1)=\mathbf{A}(\mathbf{q}) \xi(k-2 n+1) \\
& =\left[\mathbf{q}^{n}+a_{1} \mathbf{q}^{n-1}+\cdots+a_{n}\right] \xi(k-2 n+1) \\
& =\xi(k-n+1)+\cdots+a_{n} \xi(k-2 n+1) \\
& =\left[\begin{array}{llll}
1 & a_{1} & \cdots & a_{n}
\end{array}\right]\left[\begin{array}{lll}
\xi(k-n+1) & \cdots & \xi(k-2 n+1)
\end{array}\right]^{\mathrm{T}}
\end{aligned}
$$




$$
\begin{aligned}
& y(k)=\mathbf{B}(\mathbf{q}) \xi(k-n) \\
& =\left[b_{0} \mathbf{q}^{m}+b_{1} \mathbf{q}^{m-1}+\cdots+b_{m}\right] \xi(k-n) \\
& =b_{0} \xi(k-d)+\cdots+b_{m} \xi(k-n) \\
& =\left[\begin{array}{llll}
b_{0} & b_{1} & \cdots & b_{m}
\end{array}\right]\left[\begin{array}{lll}
\xi(k-d) & \cdots & \xi(k-n)
\end{array}\right]^{\mathrm{T}} \\
& \vdots \\
& y(k-n+1)=\mathbf{B}(\mathbf{q}) \xi(k-2 n+1) \\
& =\left[b_{0} \mathbf{q}^{m}+b_{1} \mathbf{q}^{m-1}+\cdots+b_{m}\right] \xi(k-2 n+1) \\
& =b_{0} \xi(k-n-d+1)+\cdots+b_{m} \xi(k-2 n+1) \\
& =\left[\begin{array}{llll}
b_{0} & b_{1} & \cdots & b_{m}
\end{array}\right]\left[\begin{array}{llll}
\xi(k-n-d+1) & \cdots & \xi(k-2 n+1)
\end{array}\right]^{\mathrm{T}}
\end{aligned}
$$

From (50)-(53) and (39) it follows that $\varphi(k)=M_{0} x(k)$, where $M_{0}$ is given by (48). It can be seen that $M_{0}$ is the $(2 n-1) \times(2 n-1)$ submatrix of $M^{\mathrm{T}}$, formed by omitting the first row and first column of $M^{\mathrm{T}}$. Note that $\operatorname{det} M_{0}=\operatorname{det} M$. Since $\mathbf{A}$ and $\mathbf{B}$ are relatively co-prime by assumption, it follows that $M$ is non-singular, and thus $M_{0}$ is non-singular.

\section{ADAPTIVE CONTROL USING RLS IDENTIFICATION}

In this section we derive a recursive least squares update law for the parameter vector $\hat{\theta}(k)$ of a controller that drives $e_{\mathrm{f}}(k+d)$ to zero asymptotically. To do this we minimize the retrospective cost function

$$
J(\hat{\theta}(k), k) \triangleq \sum_{i=d}^{k} E^{2}(\hat{\theta}(k), i)
$$

where $k \geqslant d$ and where the retrospective error is defined by

$$
E(\hat{\theta}(k), i) \triangleq y_{\mathrm{f}}(i)-b_{0} u(i-d)-\varphi^{\mathrm{T}}(i-d) \hat{\theta}(k), \quad k \geqslant d
$$

The retrospective cost function (54) defines the performance of $\hat{\theta}$ by evaluating the present value $\hat{\theta}(k)$ in terms of the past behaviour of the linear identification model (20) over the interval $d \leqslant i \leqslant k$.

Next define

$$
\begin{gathered}
\mathscr{E}(\hat{\theta}(k), k) \triangleq\left[\begin{array}{lll}
E(\hat{\theta}(k), d) & \cdots & E(\hat{\theta}(k), k)
\end{array}\right]^{\mathrm{T}} \in \mathbb{R}^{k-d+1} \\
Y(k) \triangleq\left[y_{\mathrm{f}}(d)-b_{0} u(0) \quad \ldots \quad y_{\mathrm{f}}(k)-b_{0} u(k-d)\right]^{\mathrm{T}} \in \mathbb{R}^{k-d+1}
\end{gathered}
$$


and

$$
\Phi(k) \triangleq[\varphi(0) \quad \cdots \quad \varphi(k-d)]^{\mathrm{T}} \in \mathbb{R}^{(k-d+1) \times(2 n-1)}
$$

so that

$$
\mathscr{E}(\hat{\theta}(k), k)=Y(k)-\Phi(k) \hat{\theta}(k) \in \mathbb{R}^{k-d+1}
$$

With this notation the cost function (54) can be expressed as

$$
\begin{aligned}
J(\hat{\theta}(k), k) & =\mathscr{E}^{\mathrm{T}}(\hat{\theta}(k), k) \mathscr{E}(\hat{\theta}(k), k) \\
& =[Y(k)-\Phi(k) \hat{\theta}(k)]^{\mathrm{T}}[Y(k)-\Phi(k) \hat{\theta}(k)]
\end{aligned}
$$

Next, we use a recursive least squares estimate of $\hat{\theta}(k)$ that minimizes $J(k, \hat{\theta})$, for details see, for example, Reference [14]. The RLS estimate for $\hat{\theta}(k)$ is given by

$$
\begin{gathered}
\mathscr{P}(k)=\mathscr{P}(k-1)-\frac{\mathscr{P}(k-1) \varphi(k-d) \varphi^{\mathrm{T}}(k-d) \mathscr{P}(k-1)}{1+\varphi^{\mathrm{T}}(k-d) \mathscr{P}(k-1) \varphi(k-d)}, \quad \mathscr{P}(0)>0 \\
\hat{\theta}(k)=\hat{\theta}(k-1)+\mathscr{P}(k) \varphi(k-d)\left[y_{\mathrm{f}}(k)-b_{0} u(k-d)-\varphi^{\mathrm{T}}(k-d) \hat{\theta}(k-1)\right]
\end{gathered}
$$

where the model reference adaptive control law is

$$
u(k)=-\frac{1}{b_{0}}\left[\varphi^{\mathrm{T}}(k) \hat{\theta}(k)-q^{-n+1} \mathbf{B}_{\mathrm{m}} u_{\mathrm{c}}(k)\right]
$$

\section{STABILITY OF MRAC WITH RLS IDENTIFICATION}

Define

$$
\tilde{\Theta}(k) \triangleq\left[\begin{array}{c}
\tilde{\theta}(k) \\
\vdots \\
\tilde{\theta}(k+d-1)
\end{array}\right]
$$

Then the error state vector consisting of the model matching error states and the parameter identification error states is defined by

$$
X(k) \triangleq\left[\begin{array}{c}
x_{\mathrm{e}}(k) \\
\tilde{\Theta}(k) \\
\operatorname{vec}(\mathscr{P}(k)) \\
\vdots \\
\operatorname{vec}(\mathscr{P}(k+d-1))
\end{array}\right]
$$


Using (26), (30), (60), (61) and (46), the closed-loop error dynamics with RLS identification can be represented as the $(d+1)(2 n-1)+d(2 n-1)^{2}$ dimensional system

$$
\begin{gathered}
x_{\mathrm{e}}(k+1)=A x_{\mathrm{e}}(k)-\frac{1}{b_{0}} B \varphi^{\mathrm{T}}(k) \tilde{\theta}(k) \\
\tilde{\theta}(k+1)=\tilde{\theta}(k)-\mathscr{P}(k+1) \varphi(k-d+1) \varphi^{\mathrm{T}}(k-d+1) \tilde{\theta}(k) \\
\vdots \\
\tilde{\theta}(k+d)=\tilde{\theta}(k+d-1)-\mathscr{P}(k+d) \varphi(k) \varphi^{\mathrm{T}}(k) \tilde{\theta}(k+d-1) \\
\operatorname{vec}[\mathscr{P}(k+1)]=\operatorname{vec}\left[\mathscr{P}(k)-\frac{\mathscr{P}(k) \varphi(k-d+1) \varphi^{\mathrm{T}}(k-d+1) \mathscr{P}(k)}{1+\varphi^{\mathrm{T}}(k-d+1) \mathscr{P}(k) \varphi(k-d+1)}\right] \\
\vdots \\
\operatorname{vec}[\mathscr{P}(k+d)]=\operatorname{vec}\left[\mathscr{P}(k+d-1)-\frac{\mathscr{P}(k+d-1) \varphi(k) \varphi^{\mathrm{T}}(k) \mathscr{P}(k+d-1)}{1+\varphi^{\mathrm{T}}(k) \mathscr{P}(k+d-1) \varphi(k)}\right]
\end{gathered}
$$

Note that the error system (65)-(69) is time varying since the regressor $\varphi(k)$ is a function of the exogenous signal $u_{\mathrm{c}}(k)$. Furthermore, every equilibrium of the error system (65)-(69) is of the form $\left(0, \tilde{\theta}_{q}, \mathscr{P}_{q}\right)$, where $\mathscr{P}_{q} \geqslant 0$.

\section{Remark 5.1}

Although the future parameter errors $\tilde{\theta}(k+1)$ to $\tilde{\theta}(k+d-1)$ and the future adaptation gain matrices $\mathscr{P}(k+1)$ to $\mathscr{P}(k+d-1)$ are not computed by the algorithm at time $k$, they are included in the state vector $X(k)$ to facilitate the stability analysis.

To demonstrate that every equilibrium of the system (65)-(69) is Lyapunov stable, the following lemmas are required.

\section{Lemma 5.1}

Define

$$
\begin{gathered}
V_{\mathscr{P}}(\mathscr{P}) \triangleq \operatorname{tr} \mathscr{P}^{2} \\
\Delta V_{\mathscr{P}}(k) \triangleq \operatorname{tr}\left[\mathscr{P}^{2}(k+1)-\mathscr{P}^{2}(k)\right] \\
V_{\tilde{\theta}}(\tilde{\theta}, \mathscr{P}) \triangleq \tilde{\theta}^{\mathrm{T}} \mathscr{P}^{-1} \tilde{\theta}
\end{gathered}
$$


and

$$
\Delta V_{\tilde{\theta}}(k) \triangleq \tilde{\theta}^{\mathrm{T}}(k+1) \mathscr{P}^{-1}(k+1) \tilde{\theta}(k+1)-\tilde{\theta}^{\mathrm{T}}(k) \mathscr{P}^{-1}(k) \tilde{\theta}(k)
$$

Then, for all $k \geqslant 0$,

$$
\begin{gathered}
\Delta V_{\mathscr{P}}(k) \leqslant 0 \\
\Delta V_{\tilde{\theta}}(k)=\frac{-[\varphi(k-d+1) \tilde{\theta}(k)]^{2}}{1+\varphi^{\mathrm{T}}(k-d+1) \mathscr{P}(k) \varphi(k-d+1)} \leqslant 0
\end{gathered}
$$

and $\lim _{k \rightarrow \infty} \mathscr{P}(k)$ and $\lim _{k \rightarrow \infty} \tilde{\theta}(k)$ exist.

Proof

The result follows from standard properties of RLS. See References [2, p. 60, 15, p. 202, 16, p. 22,17, p. 58].

\section{Lemma 5.2}

For all $k>0$

$$
\frac{\left[\varphi^{\mathrm{T}}(k) \tilde{\theta}(k)\right]^{2}}{1+\varphi^{\mathrm{T}}(k) \mathscr{P}(k) \varphi(k)} \leqslant \sum_{i=k}^{k+d-1} \frac{\left[\varphi^{\mathrm{T}}(i-d+1) \tilde{\theta}(i)\right]^{2}}{1+\varphi^{\mathrm{T}}(i-d+1) \mathscr{P}(i) \varphi(i-d+1)}
$$

Proof

From repeated self substitutions of (66) it follows that

$$
\begin{aligned}
\tilde{\theta}(k+d-1) & =\tilde{\theta}(k)-\sum_{i=1}^{d-1} \mathscr{P}(k+d-i) \varphi(k-i) \varphi^{\mathrm{T}}(k-i) \tilde{\theta}(k+d-i-1) \\
& =\tilde{\theta}(k)-\sum_{i=k}^{k+d-2} \mathscr{P}(i+1) \varphi(i-d+1) \varphi^{\mathrm{T}}(i-d+1) \tilde{\theta}(i)
\end{aligned}
$$

Multiply both sides of $(77)$ by $\varphi^{\mathrm{T}}(k) / \sqrt{1+\varphi^{\mathrm{T}}(k) \mathscr{P}(k) \varphi(k)}$ to get

$$
\begin{aligned}
\frac{\varphi^{\mathrm{T}}(k) \tilde{\theta}(k)}{\left[1+\varphi^{\mathrm{T}}(k) \mathscr{P}(k) \varphi(k)\right]^{1 / 2}}= & \frac{\varphi^{\mathrm{T}}(k) \tilde{\theta}(k+d-1)}{\left[1+\varphi^{\mathrm{T}}(k) \mathscr{P}(k) \varphi(k)\right]^{1 / 2}} \\
& +\sum_{i=k}^{k+d-2} \frac{\varphi^{\mathrm{T}}(k) \mathscr{P}(i+1) \varphi(i-d+1) \varphi^{\mathrm{T}}(i-d+1) \tilde{\theta}(i)}{\left[1+\varphi^{\mathrm{T}}(k) \mathscr{P}(k) \varphi(k)\right]^{1 / 2}}
\end{aligned}
$$

Now using (69) yields

$$
\begin{aligned}
\frac{\varphi^{\mathrm{T}}(k) \tilde{\theta}(k)}{\left[1+\varphi^{\mathrm{T}}(k) \mathscr{P}(k) \varphi(k)\right]^{1 / 2}}= & \frac{\varphi^{\mathrm{T}}(k) \tilde{\theta}(k+d-1)}{\left[1+\varphi^{\mathrm{T}}(k) \mathscr{P}(k) \varphi(k)\right]^{1 / 2}} \\
& +\sum_{i=1}^{k+d-2} \frac{\varphi^{\mathrm{T}}(k) \mathscr{P}(i) \varphi(i-d+1) \varphi^{\mathrm{T}}(i-d+1) \tilde{\theta}(i)}{\left[1+\varphi^{\mathrm{T}}(k) \mathscr{P}(k) \varphi(k)\right]^{1 / 2}\left[1+\varphi^{\mathrm{T}}(i-d+1) \mathscr{P}(i) \varphi(i-d+1)\right]}
\end{aligned}
$$


Using the triangle inequality and the fact that $\mathscr{P}(i) \leqslant \mathscr{P}(k)$ for all $i \geqslant k$ it follows that

$$
\begin{aligned}
\left|\frac{\varphi^{\mathrm{T}}(k) \tilde{\theta}(k)}{\left[1+\varphi^{\mathrm{T}}(k) \mathscr{P}(k) \varphi(k)\right]^{1 / 2}}\right| \leqslant & \left|\frac{\varphi^{\mathrm{T}}(k) \tilde{\theta}(k+d-1)}{\left[1+\varphi^{\mathrm{T}}(k) \mathscr{P}(k) \varphi(k)\right]^{1 / 2}}\right| \\
& +\sum_{i=k}^{k+d-2}\left|\frac{\varphi^{\mathrm{T}}(k) \mathscr{P}(i) \varphi(i-d+1)}{\left[1+\varphi^{\mathrm{T}}(k) \mathscr{P}(i) \varphi(k)\right]^{1 / 2}\left[1+\varphi^{\mathrm{T}}(i-d+1) \mathscr{P}(i) \varphi(i-d+1)\right]^{1 / 2}}\right| \\
& \times\left|\frac{\varphi^{\mathrm{T}}(i-d+1) \tilde{\theta}(i)}{\left[1+\varphi^{\mathrm{T}}(i-d+1) \mathscr{P}(i) \varphi(i-d+1)\right]^{1 / 2}}\right|
\end{aligned}
$$

Now using the Cauchy-Schwarz inequality we have

$$
\begin{aligned}
\left|\frac{\varphi^{\mathrm{T}}(k) \tilde{\theta}(k)}{\left[1+\varphi^{\mathrm{T}}(k) \mathscr{P}(k) \varphi(k)\right]^{1 / 2}}\right| \leqslant & \left|\frac{\varphi^{\mathrm{T}}(k) \tilde{\theta}(k+d-1)}{\left[1+\varphi^{\mathrm{T}}(k) \mathscr{P}(k) \varphi(k)\right]^{1 / 2}}\right| \\
& +\sum_{i=k}^{k+d-2}\left|\frac{\varphi^{\mathrm{T}}(i-d+1) \tilde{\theta}(i)}{\left[1+\varphi^{\mathrm{T}}(i-d+1) \mathscr{P}(i) \varphi(i-d+1)\right]^{1 / 2}}\right| \\
= & \sum_{i=k}^{k+d-1}\left|\frac{\varphi^{\mathrm{T}}(i-d+1) \tilde{\theta}(i)}{\left[1+\varphi^{\mathrm{T}}(i-d+1) \mathscr{P}(i) \varphi(i-d+1)\right]^{1 / 2}}\right|
\end{aligned}
$$

Lemma 5.3

Define

$$
\begin{gathered}
\Pi(k) \triangleq \operatorname{diag}\left[\mathscr{P}^{-1}(k) \quad \cdots \quad \mathscr{P}^{-1}(k+d-1)\right] \\
V_{\tilde{\Theta}}(\tilde{\Theta}, \Pi) \triangleq \tilde{\Theta}^{\mathrm{T}} \Pi^{-1} \tilde{\Theta}
\end{gathered}
$$

and

$$
\Delta V_{\tilde{\Theta}}(k) \triangleq \tilde{\Theta}^{\mathrm{T}}(k+1) \Pi^{-1}(k+1) \tilde{\Theta}(k+1)-\tilde{\Theta}^{\mathrm{T}}(k) \Pi^{-1}(k) \tilde{\Theta}(k)
$$

Then

$$
\Delta V_{\tilde{\Theta}}(k) \leqslant \frac{-[\varphi(k) \tilde{\theta}(k)]^{2}}{1+\varphi^{\mathrm{T}}(k) \mathscr{P}(k) \varphi(k)}
$$

Proof

From (63), (78) and (80) it follows that

$$
\Delta V_{\tilde{\Theta}}(k)=\sum_{i=k+1}^{k+d} \tilde{\theta}^{\mathrm{T}}(i) \mathscr{P}^{-1}(i) \tilde{\theta}(i)-\sum_{i=k}^{k+d-1} \tilde{\theta}^{\mathrm{T}}(i) \mathscr{P}^{-1}(i) \tilde{\theta}(i)
$$


Now using (75) and (76) we have

$$
\Delta V_{\tilde{\Theta}}=-\sum_{i=k}^{k+d-1} \frac{\left[\varphi^{\mathrm{T}}(i-d+1) \tilde{\theta}(i)\right]^{2}}{1+\varphi^{\mathrm{T}}(i-d+1) \mathscr{P}(i) \varphi(i-d+1)} \leqslant-\frac{\left[\varphi^{\mathrm{T}}(k) \tilde{\theta}(k)\right]^{2}}{1+\varphi^{\mathrm{T}}(k) \mathscr{P}(k) \varphi(k)}
$$

\section{Lemma 5.4}

Define

$$
V_{\Pi}(\Pi) \triangleq \operatorname{tr}\left[\Pi^{\mathrm{T}} \Pi\right]
$$

and

$$
\Delta V_{\Pi}(k) \triangleq \operatorname{tr}\left[\Pi^{\mathrm{T}}(k+1) \Pi(k+1)\right]-\operatorname{tr}\left[\Pi^{\mathrm{T}}(k) \Pi(k)\right]
$$

Then

$$
\triangle V_{\Pi}(k) \leqslant 0, \quad k \geqslant 0
$$

\section{Proof}

From (74) and (78) it follows that

$$
\begin{aligned}
\Delta V_{\Pi}(k) & =\sum_{i=k-d+1}^{k+1} \operatorname{tr} \mathscr{P}^{2}(i)-\sum_{i=k-d}^{k} \operatorname{tr} \mathscr{P}^{2}(i) \\
& =-\sum_{i=k-d}^{k} \Delta V_{\mathscr{P}}(k) \leqslant 0 \quad \square
\end{aligned}
$$

Lemma 5.5

Recall that $A(45)$ is asymptotically stable. Let $P, R \in \mathbb{R}^{n \times n}$ be positive-definite matrices that satisfy

$$
P=A^{\mathrm{T}} P A+R+I
$$

and define

$$
\sigma \triangleq \sqrt{\lambda_{\max }\left(A^{\mathrm{T} P A}\right)}
$$

Furthermore, let $\mu>0$ and define

$$
V_{x_{\mathrm{e}}}\left(x_{\mathrm{e}}\right) \triangleq \ln \left(1+\mu x_{\mathrm{e}}^{\mathrm{T}} P x_{\mathrm{e}}\right)
$$

and

$$
\Delta V_{x_{\mathrm{e}}}(k) \triangleq V_{x_{\mathrm{e}}}\left(x_{\mathrm{e}}(k+1)\right)-V_{x_{\mathrm{e}}}\left(x_{\mathrm{e}}(k)\right)
$$

Then

$$
\Delta V_{x_{\mathrm{e}}}(k) \leqslant \mu \frac{-x_{\mathrm{e}}^{\mathrm{T}}(k) R x_{\mathrm{e}}(k)+b_{0}^{-2}\left(\sigma^{2}+1\right) B^{\mathrm{T}} P B[\varphi(k) \tilde{\theta}(k)]^{2}}{1+\mu x_{\mathrm{e}}^{\mathrm{T}}(k) P x_{\mathrm{e}}(k)}, \quad k \geqslant 0
$$


Proof

Define

$$
F \triangleq \frac{1}{\sigma} P^{1 / 2} A, \quad G \triangleq \sigma P^{1 / 2} B, \quad \mathscr{J}\left(x_{\mathrm{e}}\right) \triangleq x_{\mathrm{e}}^{\mathrm{T}} P x_{\mathrm{e}}
$$

Then,

$$
\begin{aligned}
\Delta \mathscr{J}_{x_{\mathrm{e}}}(k) & \triangleq x_{\mathrm{e}}^{\mathrm{T}}(k+1) P x_{\mathrm{e}}(k+1)-x_{\mathrm{e}}^{\mathrm{T}}(k) P x_{\mathrm{e}}(k) \\
& =\left[A x_{\mathrm{e}}(k)-B \frac{\varphi(k) \tilde{\theta}(k)}{b_{0}}\right]^{\mathrm{T}} P\left[A x_{\mathrm{e}}(k)-B \frac{\varphi(k) \tilde{\theta}(k)}{b_{0}}\right]-x_{\mathrm{e}}^{\mathrm{T}}(k) P x_{\mathrm{e}}(k)
\end{aligned}
$$

Omitting the explicit dependence on $k$ we have

$$
\begin{aligned}
& \triangle \mathscr{J}_{x_{\mathrm{e}}}(k)=x_{\mathrm{e}}^{\mathrm{T}} A^{\mathrm{T}} P A x_{\mathrm{e}}-x_{\mathrm{e}}^{\mathrm{T}} A^{\mathrm{T}} P B b_{0}^{-1} \varphi^{\mathrm{T}} \tilde{\theta}-b_{0}^{-1} \varphi^{\mathrm{T}} \tilde{\theta} B^{\mathrm{T}} P A x_{\mathrm{e}}+b_{0}^{-1} \varphi^{\mathrm{T}} \tilde{\theta} B^{\mathrm{T}} P B b_{0}^{-1} \varphi^{\mathrm{T}} \tilde{\theta}-x_{\mathrm{e}}^{\mathrm{T}} P x_{\mathrm{e}} \\
& =x_{\mathrm{e}}^{\mathrm{T}}\left(A^{\mathrm{T}} P A-P\right) x_{\mathrm{e}}-x_{\mathrm{e}}^{\mathrm{T}} A^{\mathrm{T}} P B b_{0}^{-1} \varphi^{\mathrm{T}} \tilde{\theta}-b_{0}^{-1} \varphi^{\mathrm{T}} \tilde{\theta} B^{\mathrm{T}} P A x_{\mathrm{e}}+b_{0}^{-2}\left(\varphi^{\mathrm{T}} \tilde{\theta}\right)^{2} B^{\mathrm{T}} P B \\
& =x_{\mathrm{e}}^{\mathrm{T}}\left(A^{\mathrm{T}} P A-P\right) x_{\mathrm{e}}-x_{\mathrm{e}}^{\mathrm{T}} F^{\mathrm{T}} G b_{0}^{-1} \varphi^{\mathrm{T}} \tilde{\theta}-b_{0}^{-1} \varphi^{\mathrm{T}} \tilde{\theta} G^{\mathrm{T}} F x_{\mathrm{e}}+b_{0}^{-2}\left(\varphi^{\mathrm{T}} \tilde{\theta}\right)^{2} B^{\mathrm{T}} P B \\
& =x_{\mathrm{e}}^{\mathrm{T}}\left(A^{\mathrm{T}} P A-P+F^{\mathrm{T}} F\right) x_{\mathrm{e}}-x_{\mathrm{e}}^{\mathrm{T}} F^{\mathrm{T}} G b_{0}^{-1} \varphi^{\mathrm{T}} \tilde{\theta}-b_{0}^{-1} \varphi^{\mathrm{T}} \tilde{\theta} G^{\mathrm{T}} F x_{\mathrm{e}}+b_{0}^{-2}\left(\varphi^{\mathrm{T}} \tilde{\theta}\right)^{2} B^{\mathrm{T}} P B \\
& -x_{\mathrm{e}}^{\mathrm{T}} F^{\mathrm{T}} F x_{\mathrm{e}}+b_{0}^{-2}\left(\varphi^{\mathrm{T}} \tilde{\theta}\right)^{2} G^{\mathrm{T}} G-b_{0}^{-2}\left(\varphi^{\mathrm{T}} \tilde{\theta}\right)^{2} G^{\mathrm{T}} G \\
& =x_{\mathrm{e}}^{\mathrm{T}}\left(A^{\mathrm{T}} P A-P+F^{\mathrm{T}} F\right) x_{\mathrm{e}}-\left[\begin{array}{ll}
x_{\mathrm{e}}^{\mathrm{T}} & b_{0}^{-1} \varphi^{\mathrm{T}} \tilde{\theta}
\end{array}\right]\left[\begin{array}{ll}
F^{\mathrm{T}} F & F^{\mathrm{T}} G \\
G^{\mathrm{T}} F & G^{\mathrm{T}} G
\end{array}\right]\left[\begin{array}{c}
x_{\mathrm{e}}^{\mathrm{T}} \\
b_{0}^{-1} \varphi^{\mathrm{T}} \tilde{\theta}
\end{array}\right] \\
& +\left(B^{\mathrm{T}} P B+G^{\mathrm{T}} G\right) b_{0}^{-2}\left(\varphi^{\mathrm{T}} \tilde{\theta}\right)^{2} \\
& \leqslant x_{\mathrm{e}}^{\mathrm{T}}\left(A^{\mathrm{T}} P A-P+F^{\mathrm{T}} F\right) x_{\mathrm{e}}+\left(B^{\mathrm{T}} P B+G^{\mathrm{T}} G\right) b_{0}^{-2}\left(\varphi^{\mathrm{T}} \tilde{\theta}\right)^{2}
\end{aligned}
$$

Noting that

$$
F^{\mathrm{T}} F=\frac{A^{\mathrm{T}} P A}{\sigma^{2}}=\frac{A^{\mathrm{T}} P A}{\lambda_{\max }\left(A^{\mathrm{T}} P A\right)} \leqslant \frac{\lambda_{\text {max }}\left(A^{\mathrm{T}} P A\right) I_{n}}{\lambda_{\max }\left(A^{\mathrm{T}} P A\right)}=I_{n}
$$

it follows from (85) that

$$
A^{\mathrm{T}} P A-P+F^{\mathrm{T}} F \leqslant A^{\mathrm{T}} P A-P+I=-R
$$

Therefore,

$$
x_{\mathrm{e}}^{\mathrm{T}}(k)\left(A^{\mathrm{T}} P A-P+F^{\mathrm{T}} F\right) x_{\mathrm{e}}(k) \leqslant-x_{\mathrm{e}}^{\mathrm{T}}(k) R x_{\mathrm{e}}(k)
$$

which implies that

$$
\Delta \mathscr{J}_{x_{\mathrm{e}}}(k) \leqslant-x_{\mathrm{e}}^{\mathrm{T}}(k) R x_{\mathrm{e}}(k)+\left(B^{\mathrm{T}} P B+G^{\mathrm{T}} G\right) b_{0}^{-2}[\varphi(k) \tilde{\theta}(k)]^{2}
$$

Since $G^{\mathrm{T}} G=\sigma^{2} B^{\mathrm{T}} P B$, it follows from (88) that

$$
\Delta \mathscr{J}_{x_{\mathrm{e}}}(k) \leqslant-x_{\mathrm{e}}^{\mathrm{T}}(k) R x_{\mathrm{e}}(k)+\left(\sigma^{2}+1\right) B^{\mathrm{T}} P B b_{0}^{-2}[\varphi(k) \tilde{\theta}(k)]^{2}
$$


Now, since $\ln x \leqslant x-1$ for all $x>0$,

$$
\begin{aligned}
\Delta V_{x_{\mathrm{e}}}(k) & =\ln \left(1+\mu \frac{\Delta \mathscr{J}_{x_{\mathrm{e}}}(k)}{1+\mu x_{\mathrm{e}}^{\mathrm{T}}(k) P x_{\mathrm{e}}(k)}\right) \\
& \leqslant \mu \frac{x_{\mathrm{e}}^{\mathrm{T}}(k+1) P x_{\mathrm{e}}(k+1)-x_{\mathrm{e}}^{\mathrm{T}}(k) P x_{\mathrm{e}}(k)}{1+\mu x_{\mathrm{e}}^{\mathrm{T}}(k) P x_{\mathrm{e}}(k)} \\
& \leqslant \mu \frac{-x_{\mathrm{e}}^{\mathrm{T}}(k) R x_{\mathrm{e}}(k)+b_{0}^{-2}\left(\sigma^{2}+1\right) B^{\mathrm{T}} P B[\varphi(k) \tilde{\theta}(k)]^{2}}{1+\mu x_{\mathrm{e}}^{\mathrm{T}}(k) P x_{\mathrm{e}}(k)}
\end{aligned}
$$

We now present the main stability result for MRAC with RLS identification.

Theorem 5.1

Assume that the reference signal $u_{\mathrm{c}}(k)$ is bounded. Then every equilibrium of the error system dynamics (65)-(69) is Lyapunov stable, $\tilde{\theta}(k)$ and $\mathscr{P}(k)$ converge, and $y(k)-y_{\mathrm{m}}(k) \rightarrow 0$ as $k \rightarrow \infty$.

Proof

Consider the Lyapunov function candidate

$$
V(X) \triangleq a V_{x_{\mathrm{e}}}\left(x_{\mathrm{e}}\right)+V_{\tilde{\Theta}}(\tilde{\Theta}, \Pi)+V_{\Pi}(\Pi)
$$

Let $P, R \in \mathbb{R}^{n \times n}$ be positive definite and satisfy (85), and let $a>0$. Then using Lemmas 5.5, 5.3 and 5.4 it follows that

$$
\begin{aligned}
\Delta V(k) & \triangleq V(X(k+1))-V(X(k)) \\
& \leqslant a \mu \frac{-x_{\mathrm{e}}^{\mathrm{T}}(k) R x_{\mathrm{e}}(k)+\left(\sigma^{2}+1\right) B^{\mathrm{T}} P B[\varphi(k) \tilde{\theta}(k)]^{2} / b_{0}^{2}}{1+\mu x_{\mathrm{e}}^{\mathrm{T}}(k) P x_{\mathrm{e}}(k)}-\frac{[\varphi(k) \tilde{\theta}(k)]^{2}}{1+\varphi^{\mathrm{T}}(k) \mathscr{P}(k) \varphi(k)}
\end{aligned}
$$

Now from (47) it follows that

$$
\begin{aligned}
\varphi^{\mathrm{T}}(k) \mathscr{P}(k) \varphi(k) & \leqslant \varphi^{\mathrm{T}}(k) \mathscr{P}(0) \varphi(k) \\
& =x^{\mathrm{T}} M_{0}^{\mathrm{T}} \mathscr{P}(0) M x \\
& =\left(x_{\mathrm{e}}+x_{\mathrm{m}}\right)^{\mathrm{T}} M_{0}^{\mathrm{T}} \mathscr{P}(0) M_{0}\left(x_{\mathrm{e}}+x_{\mathrm{m}}\right) \\
& \leqslant x_{\mathrm{e}}^{\mathrm{T}} M_{0}^{\mathrm{T}} \mathscr{P}(0) M_{0} x_{\mathrm{e}}+x_{\mathrm{m}}^{\mathrm{T}} M_{0}^{\mathrm{T}} \mathscr{P}(0) M_{0} x_{\mathrm{m}}
\end{aligned}
$$

Let $\mu_{1}>0$ satisfy

$$
\mu_{1} P>M_{0}^{\mathrm{T}} \mathscr{P}(0) M_{0}
$$

Since, by assumption, the command signal $u_{\mathrm{c}}(k)$ is bounded and $\mathbf{A}_{\mathrm{m}}$ is stable, there exists $\beta>0$ such that

$$
x_{m}^{\mathrm{T}}(k) x_{\mathrm{m}}(k) \leqslant \beta
$$

Using (90)-(92) it follows that

$$
\begin{aligned}
\varphi^{\mathrm{T}}(k) \mathscr{P}(k) \varphi(k) & \leqslant \mu_{1} x_{\mathrm{e}}^{\mathrm{T}} P x_{\mathrm{e}}+\mu_{1} x_{\mathrm{m}}^{\mathrm{T}} P x_{\mathrm{m}} \\
& \leqslant \mu_{1} x_{\mathrm{e}}^{\mathrm{T}} P x_{\mathrm{e}}+\beta \mu_{1} \lambda_{\max }(P)
\end{aligned}
$$


Defining

$$
\mu \triangleq \frac{\mu_{1}}{1+\beta \mu_{1} \lambda_{\max }(P)}
$$

and

$$
a \triangleq \frac{b_{0}^{2}}{1+\mu_{1}\left(\sigma^{2}+1\right) B^{\mathrm{T}} P B}
$$

it follows that

$$
\begin{aligned}
\Delta V & \leqslant \frac{-\mu_{1} b_{0}^{2} x_{\mathrm{e}}^{\mathrm{T}}(k) R x_{\mathrm{e}}(k)+\mu_{1}\left(\sigma^{2}+1\right) B^{\mathrm{T}} P B[\varphi(k) \tilde{\theta}(k)]^{2}}{\left(1+\beta \mu_{1} \lambda_{\max }(P)\right)\left(1+\mu_{1}\left(\sigma^{2}+1\right) B^{\mathrm{T}} P B\right)\left(1+\mu x_{\mathrm{e}}^{\mathrm{T}}(k) P x_{\mathrm{e}}(k)\right)}-\frac{[\varphi(k) \tilde{\theta}(k)]^{2}}{1+\varphi^{\mathrm{T}}(k) \mathscr{P}(k) \varphi(k)} \\
& \leqslant \frac{-\mu_{1} b_{0}^{2} x_{\mathrm{e}}^{\mathrm{T}}(k) R x_{\mathrm{e}}(k)}{\left(1+\beta \mu_{1} \lambda_{\max }(P)\right)\left(1+\mu_{1}\left(\sigma^{2}+1\right) B^{\mathrm{T}} P B\right)\left(1+\mu x_{\mathrm{e}}^{\mathrm{T}}(k) P x_{\mathrm{e}}(k)\right)} \\
& =-\mu a \frac{x_{\mathrm{e}}^{\mathrm{T}}(k) R x_{\mathrm{e}}(k)}{1+\mu x_{\mathrm{e}}^{\mathrm{T}}(k) P x_{\mathrm{e}}(k)}
\end{aligned}
$$

Since $V(X)$ is positive definite and radially unbounded it follows from (96) that the origin of the error system (65)-(69) is Lyapunov stable. Furthermore, using Theorem A.1 it follows that $x_{\mathrm{e}}(k) \rightarrow 0$ as $k \rightarrow \infty$. Then using (32) and(35) we have that $y(k)-y_{\mathrm{m}}(k) \rightarrow 0$ as $k \rightarrow \infty$. The convergence of $\tilde{\theta}(k)$ and $\mathscr{P}(k)$ follows from Lemma 5.1.

\section{PROJECTION ADAPTIVE CONTROL ALGORITHM}

In Sections 4 and 5 we developed and proved the stability of MRAC in which the controller parameters are updated using RLS. In Sections 6 and 7 we consider the case in which the controller parameters are updated using the projection algorithm.

Consider the cost function

$$
J_{\lambda}(\hat{\theta}(k), \hat{\theta}(k-1), k) \triangleq \frac{1}{2}\|\hat{\theta}(k)-\hat{\theta}(k-1)\|_{2}^{2}+\lambda\left[y_{\mathrm{f}}(k)-b_{0} u(k-d)-\varphi^{\mathrm{T}}(k-d) \hat{\theta}(k)\right]
$$

where $\lambda$ is the Lagrange multiplier. A recursive expression for $\hat{\theta}(k)$ that minimizes (97) is given by $[2$, p. 51$]$

$$
\hat{\theta}(k)=\hat{\theta}(k-1)+\frac{\varphi(k-d)\left[y_{\mathrm{f}}(k)-b_{0} u(k-d)-\varphi^{\mathrm{T}}(k-d) \hat{\theta}(k-1)\right]}{\varphi^{\mathrm{T}}(k-d) \varphi(k-d)}
$$

Therefore, the adaptive control law is

$$
u(k) \triangleq-\frac{1}{b_{0}}\left[\varphi^{\mathrm{T}}(k) \hat{\theta}(k)-q^{-n+1} \mathbf{B}_{\mathrm{m}} u_{\mathrm{c}}(k)\right]
$$

with the parameter update

$$
\hat{\theta}(k)= \begin{cases}\hat{\theta}(k-1)+\frac{\varphi(k-d)\left[y_{\mathrm{f}}(k)-b_{0} u(k-d)-\varphi^{\mathrm{T}}(k-d) \hat{\theta}(k-1)\right]}{\varphi^{\mathrm{T}}(k-d) \varphi(k-d)}, & \varphi(k-d) \neq 0 \\ \hat{\theta}(k-1), & \varphi(k-d)=0\end{cases}
$$




\section{STABILITY OF MRAC WITH PROJECTION IDENTIFICATION}

The error state vector consisting of the model matching error states and the parameter identification error states is defined by

$$
X(k) \triangleq\left[\begin{array}{c}
x_{\mathrm{e}}(k) \\
\tilde{\Theta}(k)
\end{array}\right]^{\mathrm{T}}
$$

Using (26), (30), (46) and (100), the closed-loop error dynamics with projection identification can be represented in the state space form, for $k \geqslant 0$, by

$$
\begin{gathered}
x_{\mathrm{e}}(k+1)=A x_{\mathrm{e}}(k)-\frac{1}{b_{0}} B \varphi^{\mathrm{T}}(k) \tilde{\theta}(k) \\
\tilde{\theta}(k+1)=\left[I-\frac{\varphi(k-d+1) \varphi^{\mathrm{T}}(k-d+1)}{\varphi^{\mathrm{T}}(k-d+1) \varphi(k-d+1)}\right] \tilde{\theta}(k) \\
\vdots \\
\tilde{\theta}(k+d)=\left[I-\frac{\varphi(k) \varphi^{\mathrm{T}}(k)}{\varphi^{\mathrm{T}}(k) \varphi(k)}\right] \tilde{\theta}(k+d-1)
\end{gathered}
$$

Before presenting the main stability results we state the following lemmas.

\section{Lemma 7.1}

Define

$$
V_{\tilde{\theta}}(\tilde{\theta}) \triangleq \tilde{\theta}^{\mathrm{T}} \tilde{\theta}
$$

and

$$
\Delta V_{\tilde{\theta}}(k) \triangleq \tilde{\theta}^{\mathrm{T}}(k+1) \tilde{\theta}(k+1)-\tilde{\theta}^{\mathrm{T}}(k) \tilde{\theta}(k)
$$

Then

$$
\Delta V_{\tilde{\theta}}(k) \leqslant \frac{-\left[\varphi^{\mathrm{T}}(k-d+1) \tilde{\theta}(k)\right]^{2}}{\varphi^{\mathrm{T}}(k-d+1) \varphi(k-d+1)} \leqslant 0, \quad k \geqslant 0
$$

and, for all $t \geqslant 0,\{\|\tilde{\theta}(k+t)-\tilde{\theta}(k)\|\}_{k=0}^{\infty} \in l_{2}$.

Proof

See References [2, p. 51, 16, p. 78].

Lemma 7.2

For all $k>0$

$$
\frac{\left[\varphi^{\mathrm{T}}(k) \tilde{\theta}(k)\right]^{2}}{\varphi^{\mathrm{T}}(k) \varphi(k)} \leqslant \sum_{i=k}^{k+d-1} \frac{\left[\varphi^{\mathrm{T}}(i-d+1) \tilde{\theta}(i)\right]^{2}}{\varphi^{\mathrm{T}}(i-d+1) \varphi(i-d+1)}
$$


Proof

From repeated self substitutions of (103) it follows that

$$
\begin{aligned}
\tilde{\theta}(k+d-1) & =\tilde{\theta}(k)-\sum_{i=1}^{d-1} \frac{\varphi(k-i) \varphi^{\mathrm{T}}(k-i) \tilde{\theta}(k+d-i-1)}{\varphi^{\mathrm{T}}(k-i) \varphi(k-i)} \\
& =\tilde{\theta}(k)-\sum_{i=k}^{k+d-2} \frac{\varphi(i-d+1) \varphi^{\mathrm{T}}(i-d+1) \tilde{\theta}(i)}{\left[\varphi^{\mathrm{T}}(i-d+1) \varphi(i-d+1)\right]}
\end{aligned}
$$

Multiplying both sides of (107) by $\varphi^{\mathrm{T}}(k) / \sqrt{\varphi^{\mathrm{T}}(k) \varphi(k)}$ yields

$$
\frac{\varphi^{\mathrm{T}}(k) \tilde{\theta}(k)}{\left[\varphi^{\mathrm{T}}(k) \varphi(k)\right]^{1 / 2}}=\frac{\varphi^{\mathrm{T}}(k) \tilde{\theta}(k+d-1)}{\left[\varphi^{\mathrm{T}}(k) \varphi(k)\right]^{1 / 2}}+\sum_{i=k}^{k+d-2} \frac{\varphi^{\mathrm{T}}(k) \varphi(i-d+1) \varphi^{\mathrm{T}}(i-d+1) \tilde{\theta}(i)}{\left[\varphi^{\mathrm{T}}(k) \varphi(k)\right]^{1 / 2}\left[\varphi^{\mathrm{T}}(i-d+1) \varphi(i-d+1)\right]}
$$

The triangle inequality implies

$$
\begin{aligned}
\left|\frac{\varphi^{\mathrm{T}}(k) \tilde{\theta}(k)}{\left[\varphi^{\mathrm{T}}(k) \varphi(k)\right]^{1 / 2}}\right| \leqslant & \left|\frac{\varphi^{\mathrm{T}}(k) \tilde{\theta}(k+d-1)}{\left[\varphi^{\mathrm{T}}(k) \varphi(k)\right]^{1 / 2}}\right|+\sum_{i=k}^{k+d-2}\left|\frac{\varphi^{\mathrm{T}}(k) \varphi(i-d+1)}{\left[\varphi^{\mathrm{T}}(k) \varphi(k)\right]^{1 / 2}\left[\varphi^{\mathrm{T}}(i-d+1) \varphi(i-d+1)\right]^{1 / 2}}\right| \\
& \times\left|\frac{\varphi^{\mathrm{T}}(i-d+1) \tilde{\theta}(i)}{\left[\varphi^{\mathrm{T}}(i-d+1) \varphi(i-d+1)\right]^{1 / 2}}\right|
\end{aligned}
$$

Now using the Cauchy-Schwarz inequality we have

$$
\begin{aligned}
\left|\frac{\varphi^{\mathrm{T}}(k) \tilde{\theta}(k)}{\left[\varphi^{\mathrm{T}}(k) \varphi(k)\right]^{1 / 2}}\right| & \leqslant\left|\frac{\varphi^{\mathrm{T}}(k) \tilde{\theta}(k+d-1)}{\left[\varphi^{\mathrm{T}}(k) \varphi(k)\right]^{1 / 2}}\right|+\sum_{i=k}^{k+d-2}\left|\frac{\varphi^{\mathrm{T}}(i-d+1) \tilde{\theta}(i)}{\left[\varphi^{\mathrm{T}}(i-d+1) \varphi(i-d+1)\right]^{1 / 2}}\right| \\
& =\sum_{i=k}^{k+d-1}\left|\frac{\varphi^{\mathrm{T}}(i-d+1) \tilde{\theta}(i)}{\left[\varphi^{\mathrm{T}}(i-d+1) \varphi(i-d+1)\right]^{1 / 2}}\right|
\end{aligned}
$$

Lemma 7.3

Define

$$
V_{\tilde{\Theta}}(\tilde{\Theta}) \triangleq \tilde{\Theta}^{\mathrm{T}} \tilde{\Theta}
$$

and

$$
\Delta V_{\tilde{\Theta}}(k) \triangleq \tilde{\Theta}^{\mathrm{T}}(k+1) \tilde{\Theta}(k+1)-\tilde{\Theta}^{\mathrm{T}}(k) \tilde{\Theta}(k)
$$

Then

$$
\Delta V_{\tilde{\Theta}}(k) \leqslant-\frac{[\varphi(k) \tilde{\theta}(k)]^{2}}{\varphi^{\mathrm{T}}(k) \varphi(k)}, \quad k \geqslant 0
$$


Proof

From (63) and (109) it follows that

$$
\Delta V_{\tilde{\Theta}}(k)=\sum_{i=k+1}^{k+d} \tilde{\theta}^{\mathrm{T}}(i) \tilde{\theta}(i)-\sum_{i=k}^{k+d-1} \tilde{\theta}^{\mathrm{T}}(i) \tilde{\theta}(i)
$$

Using Lemmas 7.1 and 7.2 yields

$$
\Delta V_{\tilde{\Theta}}(k)=-\sum_{i=k}^{k+d-1} \frac{\left[\varphi^{\mathrm{T}}(i-d+1) \tilde{\theta}(i)\right]^{2}}{\varphi^{\mathrm{T}}(i-d+1) \varphi(i-d+1)} \leqslant-\frac{\left[\varphi^{\mathrm{T}}(k) \tilde{\theta}(k)\right]^{2}}{\varphi^{\mathrm{T}}(k) \varphi(k)}
$$

We now present the main stability result for MRAC with projection identification.

Theorem 7.1

Assume that the reference signal $u_{\mathrm{c}}(k)$ is bounded. Then every equilibrium of the error system dynamics (102)-(104) is Lyapunov stable, and $y(k)-y_{\mathrm{m}}(k) \rightarrow 0$ as $k \rightarrow \infty$.

Proof

The proof follows along the same lines as Theorem 5.1 except that the Lyapunov candidate is defined by

$$
V(X) \triangleq a V_{x_{\mathrm{e}}}\left(x_{\mathrm{e}}\right)+V_{\tilde{\Theta}}(\tilde{\Theta})
$$

and $\mu_{1}$ is chosen to satisfy

$$
\mu_{1} P>M_{0}^{\mathrm{T}} M_{0}
$$

where $P$ is the positive-definite matrix satisfying (85).

\section{EXAMPLE}

Example 8.1

Consider the unstable minimum phase SISO plant with relative degree $d=2$ given by

$$
\frac{y(\mathbf{q})}{u(\mathbf{q})}=\frac{\mathbf{q}+0.5}{\mathbf{q}^{3}+\mathbf{q}^{2}+\mathbf{q}+1.5}
$$

To track reference signals we choose an FIR filter as the reference model. We require $\operatorname{deg} \mathbf{A}_{\mathrm{m}}=2 n-m-1=4$ and $\operatorname{deg} \mathbf{B}_{\mathrm{m}}=\operatorname{deg} \mathbf{A}_{\mathrm{m}}-d=2$. Let the reference model be

$$
\frac{y_{\mathrm{m}}(\mathbf{q})}{u_{\mathrm{c}}(\mathbf{q})}=\frac{\mathbf{q}^{2}}{\mathbf{q}^{4}}
$$

and let $u_{\mathrm{c}}(k)$ be a square wave with period of 100 samples. The plant (113) with the control law (62) and the parameter updates (60)-(61) and (100) is simulated in MATLAB. The simulation results are shown in Figures 4 and 5. 


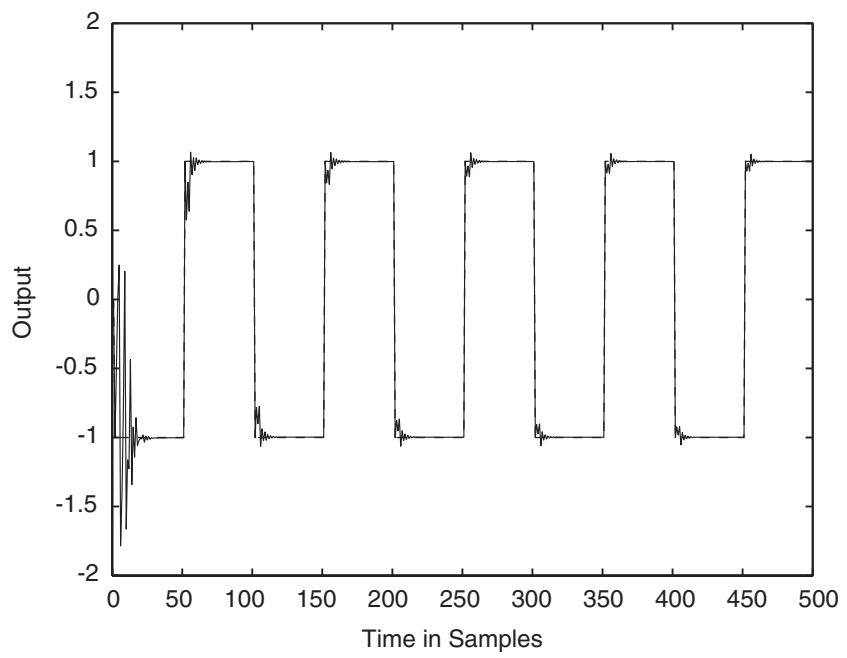

Figure 4. Tracking performance with RLS identification.

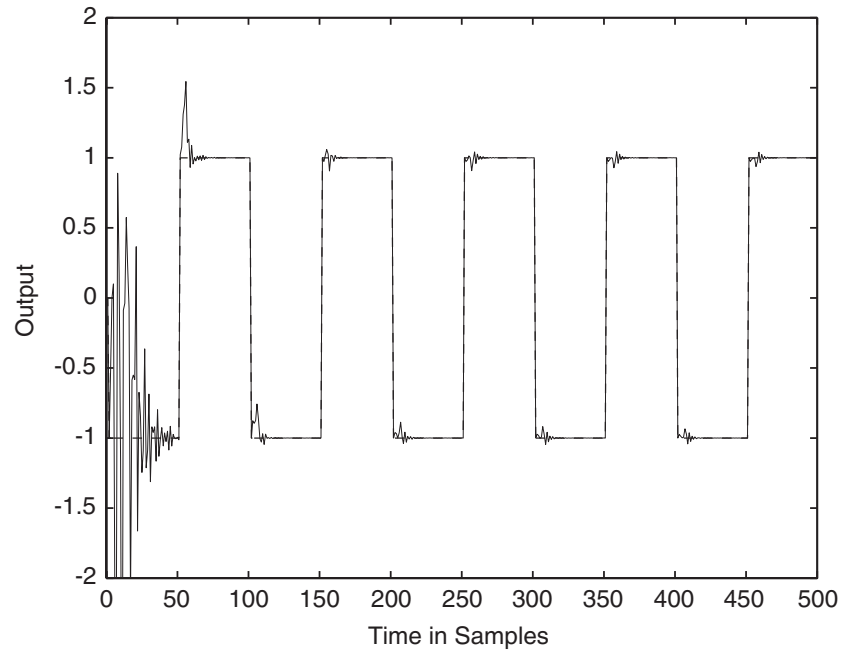

Figure 5. Tracking performance with projection identification.

\section{CONCLUDING REMARKS}

In this paper we considered model reference adaptive control using both recursive least squares and projection identification. In both cases Lyapunov stability is established. A future extension of the present work will include the supermartingale analysis of Reference [8] with alternative identification algorithms including projection, stochastic approximation, and least mean squares. 


\section{APPENDIX A}

The following result is a discrete-time version of Theorem 4.4 of Reference [18].

\section{Theorem A.1}

Let $\mathbb{N}$ denote the set of non-negative integers, let $\mathscr{D} \subset \mathbb{R}^{n}$ be an open neighbourhood of the origin, $W_{1}: \mathscr{D} \rightarrow(0, \infty), W_{2}: \mathscr{D} \rightarrow(0, \infty), W: \mathscr{D} \rightarrow[0, \infty), f: \mathbb{N} \times \mathscr{D} \rightarrow \mathbb{R}^{n}$ and $V: \mathbb{N} \times \mathscr{D} \rightarrow$ $\mathbb{R}$ be such that $W_{1}(x) \leqslant V(k, x) \leqslant W_{2}(x)$ for all $k \in \mathbb{N}$ and $x \in \mathscr{D}$ and $\Delta V \triangleq V(k+1$, $f(k, x))-V(k, x) \leqslant-W(x)$ for all $k \in \mathbb{N}$ and $x \in \mathscr{D}$. Furthermore, define $B_{r} \triangleq\{x \in \mathscr{D}:\|x\|<r\}$ and let $\tau_{r}<\min _{\|x\|=r} W_{1}(x)$. Then all solutions of $x_{k+1}=f(k, x(k))$ with $x_{0} \in\left\{x \in B_{r}: W_{2}(x) \leqslant \tau_{r}\right\}$ are bounded and satisfy $W(x(k)) \rightarrow 0$ as $k \rightarrow \infty$.

Proof

Choose $\tau_{r}>0$ such that $\tau_{r}<\min _{\|x\|=r} W_{1}(x)$. Then $\left\{x \in B_{r}: W_{2}(x) \leqslant \tau_{r}\right\} \subset\left\{x \in B_{r}: V(k, x) \leqslant \tau_{r}\right\} \subset$ $\left\{x \in B_{r}: W_{1}(x) \leqslant \tau_{r}\right\}$. Let $x_{0} \in\left\{x \in B_{r}: W_{2}(x) \leqslant \tau_{r}\right\}$. Then $x \in\left\{x \in B_{r}: V(k, x) \leqslant \tau_{r}\right\}$ for all $k \geqslant 0$. Hence $\|x(k)\|<r$ for all $k \geqslant 0$. Since $V(k, x)$ is bounded from below, the limit

$$
\lim _{k \rightarrow \infty} \sum_{\zeta=0}^{k} W(x(\zeta)) \leqslant-\lim _{k \rightarrow \infty} \sum_{\zeta=0}^{k} \Delta V(\zeta, x(\zeta))=V\left(0, x_{0}\right)-\lim _{k \rightarrow \infty} V(k, x(k))
$$

exists. This implies that $W(x(k)) \rightarrow 0$ as $k \rightarrow \infty$.

\section{REFERENCES}

1. Ioannou PA, Sun J. Robust Adaptive Control. Prentice Hall: Englewood Cliffs, NJ, 1996.

2. Goodwin GC, Sin KS. Adaptive Filtering Prediction and Control. Prentice Hall: Englewood Cliffs, NJ, 1984.

3. Goodwin GC, Ramadage PJ, Caines PE. Discrete-time multivariable adaptive control. IEEE Transactions on Automatic Control 1980; 25:449-456.

4. Becker AR, Kumar PR. Adaptive control with the stochastic approximation algorithm: geometry and convergence. IEEE Transactions on Automatic Control 1985; AC-30(4):330-338.

5. Kumar PR. Convergence of adaptive control schemes using least squares parameter estimates. IEEE Transactions on Automatic Control 1990; 35:416-424.

6. Guo L, Chen H. The Astrom-Wittenmark self tuning regulator revisited and the ELS-based adaptive trackers. IEEE Transactions on Automatic Control 1991; 36:802-812.

7. Johansson R. Global Lyapunov stability and exponential convergence of direct adaptive Control. International Journal of Control 1989; 50(3):859-869.

8. Johansson R. Supermartingale analysis of minimum variance adaptive control. Control-Theory and Advanced Technology 1995; 10(4)(Part 2):993-1013.

9. Johansson R. Lyapunov functions for adaptive systems. Proceedings of the 22nd IEEE Conference on Decision Control, San Antonio, Texas, 1983; 449-454.

10. Johansson R. Stability of direct adaptive control with RLS identification. In Modelling, Identification and Robust Control, Byrnes CI, Lindquist A (eds). North Holland: Amsterdam, 1986; 29-43.

11. Haddad WM, Hayakawa T, Leonessa A. Direct adaptive control for discrete-time non-linear uncertain dynamical systems. Proceedings of the American Control Conference, Anchorage, Alaska, 2002; 1773-1778.

12. Akhtar S, Bernstein DS. Logarithmic Lyapunov functions for direct adaptive stabilization with normalized adaptive laws. International Journal of Control 2004; 77(7):630-638.

13. Middelton RH, Goodwin GC. Digital Control and Estimation: A Unified Approach. Prentice Hall: Englewood Cliffs, NJ, 1990.

14. Astrom KJ, Wittenmark B. Adaptive Control. Addison-Wesley: Reading, MA, 1995.

15. Mosca E. Optimal Adaptive and Predictive Control. Prentice Hall: Englewood Cliffs, NJ, 1995.

16. Mareels I, Polderman JW. Adaptive Systems an Introduction. Birkhäuser: Basel, 1996.

17. Landau ID, Lozano R, M'Saad M. Adaptive Control. Springer: Berlin, 1997.

18. Khalil HK. Nonlinear Systems. Prentice Hall: Upper Saddle River, NJ, 1996. 